is estimated that these adult steers, when subjected to a 50 per cent reduction in rations consisting of hay only, went through the winter of 140 days with a loss of 1300 grams of nitrogen and approximately 52 kilograms of fat. That this loss was not inimical to the subsequent development of the steers is shown by the fact that on pasture and with concentrates and hay these animals were readily fattened for market.

The investigation here reported is being supplemented by an extensive series of observations during complete fasting. The results of the work on undernutrition are about to be published by the Carnegie Institution of Washington.

1 The results of this study were presented before the Academy on April 22, 1918. See Benedict, Miles, Roth, and Smith, Proc. Nat. Acad. Sci., 1918, 4, p. 149.

\title{
A QUANTUM THEORY OF OPTICAL DISPERSION
}

By C. G. DARWIN, F.R.S.

Normal, Bridge Laboratory of Physics, California Institute of Technology

\section{Communicated, December 1, 1922}

When a theory is framed trying to explain a discrepant system of facts, it is a necessary process of thought to take some branch of the theory as more completely true than the rest, and to adjust the remaining parts in such a way that they will fit in with this base, though they may still conflict with one another. This has certainly been true of the quantum theory; the speculations connected with it have as their base the law of the conservation of energy. Now a critical examination of fundamentals does not by any means justify this faith. It is of course a fact of observation that in the gross energy is conserved, but this only means an averaged energy; and as pure dynamics has failed to explain many atomic phenomena there seems no reason to maintain the exact conservation of energy, which is only one of the consequences of the dynamical equations. Indeed it is hardly too much to say that had the photoelectric effect been discovered a century ago, it is probable that no one would ever have suggested that the status of the first law of thermodynamics was in any way different from that of the second. On the other hand, Bohr's theory, and especially Sommerfeld's extension of it, have given great encouragement to the belief that in dynamics lay the way to the complete truth, so that in consequence of the triumphs of that theory there has been little thought in other directions. Another impediment is that our whole ideas are saturated with the principles of energy, so that denying it leaves hardly any foundation from which to start. 
Now there is another field of phenomena which forms a consistent whole, but which at present only fits into the quantum theory with a good deal of difficulty, and that is the wave theory of light. Interference and diffraction are completely explained by a wave theory, and it would seem almost impossible to devise any really different alternative which would account for them. Here is a base which seems to be free from the objections which attach to energy, and I have therefore been examining the consequences of fitting it in with those parts of the Bohr theory which seem to be most completely established. The result is what I believe to be a satisfactory theory of dispersion-one of the weakest points in the quantum theory*-and a great promise of future extensions in other directions.

We shall assume, then, that the wave theory gives a correct account of events outside matter, and it is convenient to take over the terminology of the electromagnetic theory, provided we remember that "electric force" is only to mean "light vector" and that we are not prescribing how the electric force will affect the behavior of atoms or electrons. The assumption brings with it, of course, the exact conservation of energy in the aether; it is in interchanges with matter that it need not be conserved. When a wave passes over matter there is a mutual influence and without any inquiry into what happens to the matter, we can say that it is inconceivable that the effect on the aether should be anything but in the form of an expanding spherical wave. Every such wave can be described in terms of spherical harmonics, and the simplest is the one corresponding to the harmonic of zero order. In this the electric force vanishes at two poles and is elsewhere along the lines of longitude proportional to the cosine of the latitude, while the magnetic force lies in the circles of latitude. This is the type of wave given in the classical theory by a Hertzian doublet vibrating in a line and it proves unnecessary for our theory to postulate that any more complicated type is emitted by the atom. If $x$ is the direction of the pole of the wave, then at $x, y z$ at a great distance $r$ from the atom the wave is given by:

$E_{x}=-\frac{r^{2}-x^{2}}{r^{3}} f(t-r / c), E_{y}=\frac{x y}{r^{3}} f(t-r / c), E_{z}=\frac{x z}{r^{3}} f(t-r / c)$.

Next, borrowing from the Bohr theory we shall assume that when an atom is struck by a wave, there is a certain chance that the atom should emit a secondary wave of the above type. With these assumptions it is possible to argue inductively from the observed fact that if incident waves are superposed the result can be found by an addition of their effects and from the known form of the dispersion formula. There is no need to give the argument but only its result. The complete statement of this for unpolarised 
waves is rather more complicated, but the essential points of the theory are fully represented in what follows.

When a wave, polarised so that the electric force is along $x$, strikes an atom at the origin there is a chance $A_{n}\left(\partial E_{x} / \partial t\right) d t$ that in the time $d t$ it will excite the atom to emit a spherical wave of the type (1) with $f$ of the form $\alpha_{n} e^{-\lambda_{n} t} \cos k_{n} t$. Here $A_{n}, \alpha_{n} \lambda_{n}$ and $k_{n}$ depend only on the nature of the atom and not at all on the incident force. $\lambda_{n}$ is supposed to be small. Of course $\partial E_{x} / \partial t$ may be negative; in this case we shall suppose that there is a chance $A_{n}\left(-\partial E_{x} / \partial t\right) d t$ for the emission of a wave $-f$. We shall be able to treat both cases together and need not make the distinction. The subscript $n$ indicates that we suppose there are several different ways in which the atom may be excited, each with a separate chance for it.

Consider a simple case, a monochromatic wave polarised along $x$ and advancing along $z$, which strikes a group of $N$ atoms at the origin. Let the wave be $E_{x}=H_{y}=F \cos p(t-z / c)$. The number excited in the interval $d t$ will be $N A_{n}(-F p \sin p t) d t$. Consider the secondary wave crossing the point $x, y, z$ at the time $t+r / c$. This is due to all the atoms which were excited before the time $t$. The number excited in the interval $d s$ at a time $t-s$ is $N A_{n}(-F p \sin p(t-s)) d s$ and each of these will at the time $t$ be giving a wave typified by $f=\alpha_{n} e^{-\lambda_{n} s} \cos k_{n} s$. So that total effect will be a wave which at the time $t+r / c$ at $x, y, z$ has $x$-component

$$
\begin{aligned}
E_{x} & =-N A_{n} F p\left(-\frac{r^{2}-x^{2}}{r^{3}}\right) \int_{0}^{\infty} \sin p(t-s) d s . \alpha_{n} e^{-\lambda_{n} s} \cos k_{n} s(2) \\
& =N A_{n} \alpha_{n} \frac{r^{2}-x^{2}}{r^{3}} F \frac{p^{2}}{k^{2}-p^{2}} \cos p t
\end{aligned}
$$

provided that $\lambda_{n}$ is taken as small. The averaging has entirely blotted out the frequency of the atoms and left only that of the incident wave. Now on the classical theory, if there is a group of $N_{n}$ electrons which have a natural frequency of vibration $k_{n} / 2 \pi$, the wave they scatter is given by

$$
E_{x}=N_{n} \frac{e^{2}}{m c^{2}} \frac{r^{2}-x^{2}}{r^{3}} F \frac{p^{2}}{k^{2}-p^{2}} \cos p t .
$$

So if we identify $N_{n} e^{2} / m c^{2}$ with $N A_{n} \alpha_{n}$ the expressions are the same. But the only difference between the phenomenon of scattering and of the refractive index lies in the matter of allowing for the mutual influence of the atoms, an influence exerted by the waves they send out and therefore the same on both theories. So we may at once say that from our result will follow the dispersion formula of Lorentz

$$
\frac{3\left(\mu^{2}-1\right)}{\mu^{2}+2}=\Sigma_{n} \frac{4 \pi N A_{n} \alpha_{n} c^{2}}{k_{n}^{2}-p^{2}} .
$$


From the linear way in which the chance of excitation depends on the incident force, it follows that the average effects of superposed waves is additive; in other words the atoms act as Fourier analysers, sort out the harmonic components of an arbitrary incident wave and refract each component in the proper degree. In all cases the characteristics frequency with which the waves are really emitted will entirely disappear by averaging.

It will be necessary to consider the balance of energy which is nearly but not quite exact, but the present simple equations are not suited for this; they fail to give the balance even in the classical case, and there it must occur. This question is better treated in connection with absorption. The problem is complicated by the fact that the excited wave may possibly have a phase differing slightly (it may only be slightly) from that of a cosine. I have assumed the form of the damping factor as $e^{-\lambda_{n} t}$ only for convenience; all that is necessary is that the infinite end should be unimportant. An alternative is to suppose that the wave is undamped but that there is a chance $\lambda_{n} d t$ in every element of time $d t$ that it should stop. We have only discussed waves polarised along the $\dot{x}$-axis and have supposed that the excited waves have this axis as pole; for the general case the formulation must be somewhat changed, but it would take too long to state and prove the modification here. The essential points of the theory are not altered, and it also appears that there should be no particular difficulty in fitting double refraction and rotatory dispersion into our scheme.

A theory of dispersion is not of course complete without including selective absorption. If $\lambda_{n}$ is retained in the integration of (2) the result is an expression practically the same as that given in the classical theory when a damping factor is included. Observe that on the present theory when the forced period approaches the natural, there is no increase either in the number of atoms excited or in the strength of the waves they send out. The whole change is due to the greater efficiency with which they reinforce the primary beam. Our theory gives no explanation of the mechanism of conversion of radiant energy into atomic heat, any more than does the classical theory with its damping factor. The conversion is probably better studied by the consideration of other cases of absorption, such as metallic reflexion, and our method of argument, applied to this last, should certainly give interesting results. We shall have to find what emission of spherical waves will diminish the aethereal energy when superposed on the incident wave. Thus a wave like that for dispersion would do for metallic reflexion, if the phase is suitably altered, or possibly we may suppose that the wave is again in the form of a cosine, but that the chance of excitation is now proportional to $E_{x}$ instead of to $\partial E_{x} / \partial t$. It seems likely that a study of the optical constants of metals would throw light on this question. Afterwards it would be necessary to examine the 
balance of energy between aether and matter and this might help in understanding the mechanism of the process.

We may now review how these speculations will modify the accepted theory. As we have made no assumptions as to what goes on inside the atom, we can take over the whole of the dynamics of stationary states. We suppose that an atom is usually in its lowest quantum state. The motions of the electrons will sometimes lead to a favourable configuration and when this occurs in the presence of a changing electric force there is a chance that the atom may be jerked into a condition in some way associated with one of its higher quantised states. It at once starts radiating with a frequency corresponding to the return from that state to the lowest. Dispersion throws no light on the amplitude of the wave, for in the formula it always occurs multiplied by the probability factor $A_{n}$. It is rather tempting to suppose that it actually goes into the higher quantised state, and then gives a wave of such amplitude and length that, but for the interference with the incident light, it would emit energy $h k_{n} / 2 \pi$. If this is so we may perhaps extend our theory to cover pure emission; for though we have not postulated any precise relationship between electric force and electrons, it seems inevitable that there should be a rapidly changing electric force near a moving electron, and this force would have a chance of jerking the atom into its higher state. On the other hand difficulties are raised in other directions. For the rediation must be immediate and therefore the state would not really be stationary at all, and the accepted theory of specific heats requires that a molecule should be able to remain in its higher states. In any case there is a clear contradiction to the principle of energy, but the phases of the outgoing waves are so adjusted that for cases of pure scattering or refraction on the average as much energy goes out as comes in.

There are many other points that will require attention. In the first place the refractive index is closely related to the dielectric constant. Now though it is quite proper to treat the dielectric constant as a limiting case of refraction yet it can be regarded electrostatically and it will be necessary to see the physical meaning of this aspect. Again it is possible to count the electrons in the atoms by $\mathrm{X}$-ray reflexion, and it follows that there must be a relation between the $e^{2} / m c^{2}$ of the classical theory and our $A_{n} \alpha_{n}$. In this connection I owe to Prof. P. S. Epstein the suggestion that the theory may explain the defect observed in the scattering of hard $\gamma$-rays below that predicted. Here the wave length of the incident light is much shorter than the distances between the electrons and the incoherent waves cannot recombine in the way they do under the classical theory. Lastly, it will be necessary to reëxamine the deduction of the formula for black radiation, for all present proofs are founded on theorems following out of the conservation of energy. 
In view of the great number of problems that are suggested and the probability that it will take a considerable time to deal with them, it appeared to me that it might be of interest to publish this preliminary account of a very incomplete theory.

* The difficulty is that the standard theory indicates a dispersion formula involving the frequency of the electron's motion in the atom, which is quite different from its absorption frequency.

\section{CIRCUS MOVEMENTS OF LIMULUS}

\section{By William H. Cole}

\section{LAKE FOREST COLLEGE}

Communicated, December 4, 1922

By the symmetric and asymmetric stimulation by light of Limuli (from 20 to $60 \mathrm{~mm}$. in diameter) it has been demonstrated that the animals are positively phototropic and that they show positive circus movements. It has further been found that the diameter of the circles turned as the result of asymmetric stimulation is inversely proportional to the light intensity. Because of the importance of circus movements in the analysis of orientation to light, and because there has recently appeared in these pages by Professor Mast a new explanation of orientation in insects, opposed to the tropism theory, the experiments leading to the above conclusions are briefly reported at this time. A fuller account will appear later in another journal.

In all the experiments on circus movements with Limuli the conditions were such that the animals were subjected to diffuse and non-directive illumination, the only conditions under which circus movements should ever be investigated. This was accomplished by arranging several Mazda lamps around the outside of a cylindrical glass dish lined on the inside with tissue paper. Three intensities of light were obtained by placing the lamps at different distances from the center of the dish, viz., 150, 300 and $450 \mathrm{~mm}$., and although the intensities were not accurately determined, they were always in the same proportion, and were approximately 8000 , 2000 and 900 candle meters. The conditions of asymmetry in the photoreceptors were produced either by removing or by blackening the two median eyes and one lateral eye, leaving the other lateral eye functional. One hundred thirty-five experiments on thirty-eight animals furnished the data presented here. The paths of the animals were transferred to record sheets, and were afterward measured as to length and number of degrees" turned per centimeter. From table $I$ it is seen that the number of degrees 